\title{
NSAIDs and heart failure: $A$ dangerous relationship
}

\author{
Raffaele Rotunno', Igino Oppo1, Gabriele Saetta1, Pietro Aveta1, Sergio Bruno2 \\ 1 Cardiology Department CCU, Roccadaspide Hospital, Salerno, Italy \\ 2 ASL, Salerno, Italy
}

\begin{abstract}
One of the potential cardiotoxic action of anti-inflammatory drugs is the occurrence of heart failure (HF), due to their effects on fluid retention and blood pressure. The risk of hospitalization for $\mathrm{HF}$ is roughly doubled for both Coxibs, cyclooxygenase-1 (COX-1) and cyclooxygenase-2 (COX-2) inhibitors, and all the conventional nonsteroidal anti-inflammatory drugs (NSAIDs). These drugs are also associated with a risk of vascular thrombosis, which for NSAIDs is different in relation to their different ability to inhibit COX-1 and COX-2.

The cardiovascular toxicity of these drugs in the direction of $\mathrm{HF}$ follow different pathways respect to their related vascular thrombosis toxicity and involves, in particular, the renal prostaglandins, $\mathrm{PGE}_{2}$ and prostacyclin, mostly synthesized by COX-2.

In the kidneys the PGs perform a direct vasodilatory action, e.g. by means of non-contrasting angiotensin mechanisms, and for this reason nimesulide effects on renal microcirculation are independent from the prevalence of intrarenal renin angiotensin aldosterone system (RAAS) activity. Conversely, nimesulide reduces sodium tubular urinary flow only in presence of intrarenal RAAS.
\end{abstract}

\section{Importance of the issue and epidemiological data}

A potential cardiotoxic action of anti-inflammatory drugs is the $\mathrm{HF}$ due to their effects on fluid retention [1] and blood pressure [2]. Observational studies have shown association between the use of NSAIDs and the occurrence or the exacerbation of HF. In the late 90s Heerdink et al. [3] found that patients treated with a diuretic during the three weeks following NSAIDs therapy were more likely hospitalized for HF. Two years later Page and Henry [4] in a case-control study noticed that

Corresponding author: Raffaele Rotunno, Cardiology Department CCU, Roccadaspide Hospital, Salerno, Italy. Tel. 08289426227.

E-mail: raffaele.rotunno@cheapnet.it

Key words: Non-steroidal anti-inflammatory drugs; heart failure; prostaglandins.

Received for publication: 24 April 2018

Accepted for publication: 11 May 2018

(C) Copyright R. Rotunno et al., 2018

Tipografia PI-ME Editrice, Italy

Monaldi Archives for Chest Disease 2018; 88:950

doi: 10.4081/monaldi.2018.950

This article is distributed under the terms of the Creative Commons Attribution Noncommercial License (by-nc 4.0) which permits any noncommercial use, distribution, and reproduction in any medium, provided the original author(s) and source are credited. the use of NSAIDs in the last two weeks of therapy doubled the chances of being hospitalized for HF; in particular, the risk of HF increased dramatically in the presence of heart disease. Subsequently, Feenstra et al. [5] studied the relationship between NSAIDs and the first occurrence of $\mathrm{HF}$ or exacerbation in the elderly people, enrolled in the Rotterdam study. According to these authors, NSAIDs are likely to precipitate a relapse of HF, but less frequently they are responsible for its first event. In this study the current use of anti-inflammatory drugs, while was not significantly related to an increase of the first episodes of HF-related hospitalization, increased by approximately 4 times the risk of exacerbation of chronic HF. However, where there was a first manifestation of HF during NSAID treatment, usually the presence of systemic arterial hypertension (SAH) or coronary artery disease (CAD) occurred, conditions that make renal and cardiovascular homeostasis more susceptible to prostaglandins (PGs). This was confirmed by Huerta [6] several years later in another case-control study conducted in the general population of U.K. from January 1997 to December 2000; an history of SAH, diabetes mellitus and kidney failure made patients more vulnerable to NSAIDs-related cardiotoxicity. Moreover, the addition of NSAIDs to the therapy increased the risk of first hospitalization for HF already in the first month in all the hypertensive patients except those, who adopted a calcium channel blocker.

In a similar study [7] the highest risk was observed in patients receiving NSAIDs, while being treated with an angiotensin converting enzyme (ACE) inhibitor, confirming the pharmacodynamic interaction of the two drugs at renal level [8].

In the 2008 a meta-analysis of observational studies and randomized clinical trials on the relationship between anti-inflammatory drugs and HF was published [9]. Both the observational studies and the metaanalysis showed a significant increase in the risk of HF in the subjects treated with conventional NSAIDs or Coxibs, particularly in those with joint disease. In the 2009 a case-control study [10] emphasized the relationship between the risk of $\mathrm{HF}$ and the single anti-inflammatory drugs, observing that the risk of HF changes according to the drug used: higher for etoralac and etoricoxib, while almost absent for celecoxib. While the only use of Coxibs and diclofenac increased the risk of death in patients with chronic HF, the consumption of all anti-inflammatory drugs, conventional NSAIDs and Coxibs, increased the likelihood of developing a non-fatal myocardial infarction.

In the 2013, following these alarming data coming from studies on Coxibs and traditional NSAIDs, CNT Collaborative Group published a meta-analysis of randomized clinical trials [11], which sought to quantify, at the same time, the anti-inflammatory drugs-related cardiovascular and gastrointestinal risk. The meta-analysis showed that the risk of hospitalization for HF is roughly doubled for both the Coxibs and all the conventional NSAIDs studied. However, these drugs are also associated with a risk of vascular thrombosis; for NSAIDs is different in relation to their different ability to inhibit the two cyclooxygenases (COX-1, which produces the thromboxane and COX-2, which produces the prostacyclin, antagonist of thromboxane). In this regard, the meta-analysis indicates that Coxibs and diclofenac, among the traditional NSAIDs, increase of about $1 / 3$ the major thrombotic vascular events. The risk of thrombosis, associated with ibuprofen, is 
largely absent for naproxen. Conversely, all the anti-inflammatory drugs, included naproxen, increase the likelihood of hospitalization for HF by almost doubling it. Accordingly, the cardiovascular toxicity of these drugs in the direction of HF follow different pathways respect to their related vascular thrombosis toxicity and involves, in particular, the renal prostaglandins, PGE2 and prostacyclin, mostly synthesized by COX-2, which is exceptionally present in the kidney in constitutive form.

\section{Renal responses to COX2 inhibition in the presence of different intrarenal Ang II activity}

The role of PGs in controlling the flow of renal blood during the acute fall of cardiac output was demonstrated by Oliver et al. in 1981 [12].

When in the 17 anesthetized dogs the venous return to the heart was reduced by inflating a ball in the thoracic inferior vena cava, a decrease of the cardiac output was observed with an increase of the total peripheral vascular resistance, without altering the renal ones. Therefore, only a negligible drop in the renal flow was observed.

However, because plasma renin activity and catecholamine concentrations were both markedly high at systemic and renal levels, also systemic and renal resistance would have been increased simultaneously. On the contrary, instead, at renal level vascular resistance was not changed and blood flow was preserved as a result of the increased prostaglandin synthesis, which counteract the vasoconstrictive action of angiotensin and noradrenaline.

According to some works it appears that, while angiotensin II exerts its vasoconstrictor effect on intrarenal efferent arteriole, PGs exert their vasodilatory effect on the afferent arteriole (Brown-Herchuelz), thus optimizing the glomerular perfusion pressure.

Subsequently, the anesthetized dogs were treated with indomethacin to reduce at renal level PGE2 concentrations. Thereby, the obstacle to the action of angiotensin and noradrenaline was removed and the vascular resistance increased, especially at the afferent arteriole level, reducing kidneys blood flow.

Significant variations in PGE synthesis in response to angiotensin renin activation were detectable at renal level, but not at systemic level. Consequently, the damage induced by NSAIDs is primarily localized at renal level.

When the cardiac output impairment is permanent, as in patients with chronic HF, the plasma values of renin activity and prostaglandin metabolites changes in relation to plasma sodium concentration, namely they both inversely correlate with blood sodium [1].

The lower the blood sodium values, the higher the levels of angiotensin and aldosterone, and the greater is the vasodilatory PGs production, whose contrast role at renal level becomes even more precious.

In these subjects NSAIDs, by inhibit PGs renal synthesis through COX-2 blocking, make undisputed the renal vasoconstriction and the anti-diuretic action, produced by RAAS's exalted activity, with the increasing of renal vascular resistance and impaired diuresis.

The result at systemic level is a significant increase in ventricular filling pressures, which is a catastrophic event in a subject with precarious hemodynamic balance.

It should be noted that, due to their vasodilatory action and to the inhibition of tubular sodium reabsorption, renal PGs are primarily responsible for natriuresis. COX-2 is responsible for the synthesis of PGE2 and PGI, which exert their vasodilatory effect in both the cortex and the renal medulla.

A different scenario is observed with regard to sodium reabsorption: COX-2 is expressed in the cortex at the dense macula level, where through the synthesis of PGE2 regulates renin release by juxtaglomerular cells with an antinatriuretic effect, and, contemporarily, in the medulla at an interstitial level, where through the synthesis of PGE2 counteracts the absorption of aldosterone-dependent water and salt with natriuretic effect.

In an experimental work of Torrance Green Sprague-Dawley anesthetized rats were treated with nimesulide, a selective COX-2 inhibitor [13]. To increase cortical COX-2 expression - tubuloglomerular feedback and renin release via PGE2- Sprague-Dawley anesthetized rats were kept in sodium restriction or under captopril treatment.

In presence of nimesulide a similar renal flow reduction was observed in sodium restriction rats, therefore with high levels of angiotensin, and in rats receiving captopril, then with low angiotensin levels.

Conversely, a reduction in urinary sodium excretion was observed in salt restriction rats, therefore with high levels of aldosterone, but not in rats treated with an ACE-I, hence with low aldosterone levels.

In conclusion, PGs exert a direct renal vasodilatory action, i.e. through mechanisms that do not contrast to angiotensin, and, therefore, nimesulide effects on renal microcirculation are not dependent from RAAS activity prevalence. Conversely, nimesulide reduces sodium urinary flow only in the presence of intranasal RAAS activation.

It is well known, in fact, that aldosterone is the main regulator of the epithelial sodium channel alpha subunit (alphaENaC) at collector duct level, increasing the mRNA synthesis of that channel.

Prostanoid EP1 receptor activation, present in the medullary collector ducts, by the PGE2, COX-2 dependent, stanches the over-regulation of alfaENaC mRNA synthesis, induced by aldosterone, hampers the synthesis of alphaENaC channels increase and, consequently, prevents RAAS-dependent sodium reabsorption [14].

In the event that $\mathrm{Na}$ tubular reabsorption through alphaENaC is removed with an ACE-I, nimesulide mediated $\mathrm{COX}_{2}$ blocking has less relevant effects.

\section{Conclusions}

A recent review of seven studies - 4 case/control studies and 3 cohort studies - [15] confirmed that patients using NSAIDs have a risk of $17 \%$ higher of developing $\mathrm{HF}$.

In August 2016, the American Heart Association (AHA) has recognised, with evidence of grade B, Coxibs and NSAIDs as drugs potentially able to induce $\mathrm{HF}$ following water and sodium retention, increase the systemic vascular resistance and compromised the response to diuretics (Circulation 2016;134).

\section{References}

1. Dzau VJ, Packer M, Lilly LS, et al. Prostaglandins in severe congestive heart failure. Relation to activation of the renin-angiotensin system and hyponatremia. N Engl J Med 1984;310:347-52.

2. Wilson SL, Poulter NR. The effect of non-steroidal anti-inflammatory drugs and other commonly used non-narcotic analgesics on blood pressure level in adults. J Hypertens 2006;24:1457-69.

3. Heerdink ER, Leufkens HG, Herings RM, et al. NSAIDs associated with increased risk of congestive heart failure in elderly patients taking diuretics. Arch Intern Med 1998;158:1108-112.

4. Page J, Henry D. Consumption of NSAIDs and the development of congestive heart failure in elderly patients: an underrecognized public health problem. Arch Intern Med 2000;160:777-84. 
5. Feenstra J, Heerdink ER, Grobbee DE, Stricker BH. Association of nonsteroidal anti-inflammatory drugs with first occurrence of heart failure and with relapsing heart failure: the Rotterdam Study. Arch Intern Med 2002;162:265-70.

6. Huerta C, Varas-Lorenzo C, Castellsague J, García Rodríguez LA. Non-steroidal anti-inflammatory drugs and risk of first hospital admission for heart failure in the general population. Heart 2006;92:1610-5.

7. Garcia Rodriguez LA, Hernadez-Diaz S. Non steroidal antiinflammatory drugs as a trigger of clinical heart failure. Epidemiology 2003;14:240-6.

8. Green T, Gonzalez AA, Mitchell KD, Navar LG. The complex interplay between COX-2 and angiotensin II in regulating kidney function. Curr Opin Nephrol Hypertens 2012;21:7-14.

9. Scott PA, Kingsley GH, Scott DL. Non-steroidal anti-inflammatory drugs and cardiac failure: meta-analyses of observational studies and randomised controlled trials. Eur J Heart Fail 2008;10:1102-7.

10. Gislason GH, Rasmussen JN, Abildstrom SZ, et al. Increased mortality and cardiovascular morbidity associated with use of nonsteroidal anti-inflammatory drugs in chronic heart failure. Arch Intern Med 2009;169:141-9.

11. Coxib and traditional NSAID Trialists' (CNT) Collaboration. Vascular and upper gastrointestinal effects of non-steroidal antiinflammatory drugs: meta-analyses of individual participant data from randomised trials. Lancet 2013;382:769-79.

12. Oliver JA, Sciacca RR, Pinto J, Cannon PJ. Participation of the prostaglandins in the control of renal blood flow during acute reduction of cardiac output in the dog. J Clin Invest 1981;67:229-37.

13. Green T, Rodriguez J, Navar LG. Augmented cyclooxygenase-2 effects on renal function during varying states of angiotensin II. Am J Physiol Renal Physiol 2010;299:F954-62.

14. González AA, Céspedes C, Villanueva S, et al. Prostanoid-1 receptor regulates renal medullary $\mathrm{aENaC}$ in rats infused with angiotensin II. Biochem Biophys Res Commun 2009;389:372-7.

15. Ungprasert P, Srivali N, Thongprayoon C. Nonsteroidal antiinflammatory drugs and risk of incident heart failure: A systematic review and meta-analysis of observational studies. Clin Cardiol 2016;39:111-8. 